\title{
The water-eluting component of Anemarrhena asphodeloides Bge. laxative effects on loperamide- induced constipation rats
}

xiaomao li

Heilongjiang University of Chinese Medicine

Yan Liu

Heilongjiang University of Chinese Medicine

Yanping Sun

Heilongjiang University of Chinese Medicine

Yonggang Xia

Heilongjiang University of Chinese Medicine

Bingyou Yang ( $\sim$ ybywater@163.com )

Heilongjiang University of Chinese Medicine https://orcid.org/0000-0002-2310-2750

Haixue Kuang

Heilongjiang University of Chinese Medicine

\section{Research}

Keywords: Constipation, Loperamide, treament, ICCs, AQPs

Posted Date: September 3rd, 2020

DOl: https://doi.org/10.21203/rs.3.rs-67586/v1

License: (c) (i) This work is licensed under a Creative Commons Attribution 4.0 International License.

Read Full License 


\section{Abstract \\ Background}

Slow transit functional constipation (STC) is the most common type of chronic constipation, with seriously affecting people's survival and quality of life in the recent years. Based on the safety and small side effects of traditional Chinese medicine, it is urgent to research and develop new medicine for the treatment of constipation. As a traditional Chinese medicine, Anemarrhena asphodeloides Bge. has been reported treat constipation in a long time, and carbohydrate is its active ingredient.

\section{Methods}

ELISA used for detection of serum gastrointestinal hormones and neurotransmitters levels. HE stain was analyzed to evaluate the colon injury. Western blot and RT-PCR detected interstitial cells of Cajal markers (SCF/c-Kit) and aquaporins (AQPs).

\section{Results}

The mainly compositions of oligosaccharide and amino acid from AABW was determined by HPLC. Based on the laxative activities of AABW in the study, AABW obviously enhanced the contents of VIP, Gas, MTL, SP and 5-HT and decreased the levels of NO from loperamide-induced rats by Elisa kits. Additionally, AABW could up-regulate the expressions of SCF, c-Kit, AQP3, VIP and down-regulate AQP8 to repair the damage of colon.

\section{Conclusion}

These findings suggested that $A A B W$, which could treat constipation through mediating intestinal motility and water metabolism, were laxative active constituents from Anemarrhena asphodeloides Bge..

\section{Background}

Slow transit functional constipation (STC) is the most common type of chronic constipation, and also a frequent clinical disease. It is often a functional bowel disorder characterized by persistent hardship, reduced frequency of defecation or a feeling of endless defecation [1-3]. With the changes of human lifestyle and life structure, the incidence of STC is on the rise, and it has seriously affected people's survival and quality of life in the recent years $[4,5]$. The pathophysiology of constipation is not well understood, but it has been suggested that the pathogenesis of constipation is likely to be multi-factor, resulting from inflammation, secretory dysfunction, gastrointestinal motility disorders, and changes in gastrointestinal innervation $[6,7]$. To date, a range of traditional and modern medicines, including Senokot, Correctol, Dulcolax and Tegacero, were often used to treat constipation because of their 
cathartic effects. These drugs increase the contraction of the smooth muscles of the gut, promote gastrointestinal motility and promote defecation. However, high costs and adverse reactions limit their use in the treatment of constipation. Prokinetic agents, especially Tigacelo, are still commonly used today as laxatives, but are prone to life-threatening coronary artery constriction and myocardial infarction [8]. Based on the safety and small side effects of traditional Chinese medicine, it is urgent to research and develop new medicine for the treatment of constipation.

As a traditional Chinese medicine, Anemarrhena asphodeloides Bge. has been reported various pharmacological effects including senile dementia improvement, anti-tumor, anti-platelet aggregation, anti-osteoporosis, anti-inflammation, antibiosis and antidiabetics [9]. There is increasing evidence to indicate that it has potential for use in the treatment of constipation. It is reported that polysaccharides of Anemarrhena asphodeloides treat constipation via enhancing gastrointestinal motility [10]. However, the precise effects on the water-eluting component of Anemarrhena asphodeloides Bge. (AABW) on constipation have not been investigated thus far, at least to the best of our knowledge.

In this study, AABW was studied to explore the effects and potential mechanism for the laxative treatment, by evaluating the basic indicators of faeces number, faeces wet weight water content, and some futher characteristics of the intestinal motility, muscle abnormality, intestinal nervous system disorder, intestinal stromal cell abnormality and water channel abnormality. The above researches could make some contributions to the development of healthy and non-toxic laxatives.

\section{Materials And Methods}

\subsection{Materials}

Loperamide was obtained from Sigma chemicals Co (MO, Louis, USA). The remaining chemicals were of analytical grade.

\subsection{Plant material collection and HPLC analysis}

The rhizomes of Anemarrhena asphodeloides Bge. were collected from Hebei province of China in 2016. After botanical identification by comparative macroscopic and microscopic studies by A/Prof. Ruifeng Fan (Medicinal Botany, Heilongjiang University of Chinese Medicine), the specimens (No.20160901) were identified as the rhizomes of Anemarrhena asphodeloides Bge. (Liliaceae) and deposited in the herbarium of the Department Chinese Medicine Chemistry of Heilongjiang University of Chinese Medicine.

The dried Anemarrhena asphodeloides rhizomes $(1 \mathrm{~kg})$ were fractured into pieces and extracted three times with boiling water $(1: 10, \mathrm{wt} / \mathrm{wt})$ for $3 \mathrm{~h}$. The combined extracts were centrifuged to remove impurities. The supernatant was concentrated, precipitated with $95 \%$ ethanol and stored at $4{ }^{\circ} \mathrm{C}$ overnight. After centrifugation ( $3000 \mathrm{rpm}$ for $10 \mathrm{~min}$, at $25^{\circ} \mathrm{C}$ ), the supernatant was vacuum-dried and reserved at $4{ }^{\circ} \mathrm{C}$ until use. The supernatant was subjected to column chromatography on a D101 type macroporous adsorption resin, and eluted with water, $20 \%$ ethanol, $60 \%$ ethanol, and $95 \%$ ethanol in turn. Water eluting 
component was obtained and determined by high performance liquid chromatography (HPLC) analysis and identify the composition. The water eluting fraction was dissolved in water at a concentration of $1.00 \mathrm{mg} / \mathrm{mL}$. HPLC analysis was performed on a Atlantis T3 $(4.6 \mathrm{~mm} \times 250 \mathrm{~mm}, 3 \mathrm{~mm})$ maintained at $30^{\circ} \mathrm{C}$, the mobile phase $A$ was water and the mobile phase was methanol. the total run time was 30 min the gradient was: $0-30 \mathrm{~min}(\mathrm{~A}: 95 \%-0 \%)$ The injection volume was $10 \mathrm{~mL}$ and the flow rate was set at $1.0 \mathrm{~mL} / \mathrm{min}$. Detection wavelength was set at $254 \mathrm{~nm}$. Figure 1 showed the HPLC chromatograms of the fructose samples, the content of fructose detected by HPLC-UV analysis was more than $90 \%$.

\subsection{Experimental animals}

Adult Wistar rats (male, $200 \pm 20$ g, single cage breeding, Certificate: SDXK (Liao) 2017-0065) were purchased from Harbin Medical University. All animal experiments were implemented according to the Animal Care Committee of the School of Medicine, Heilongjiang University of Chinese Medicine (Approval Number, 2017050801). Rats were housed under a constant temperature of $25 \pm 2{ }^{\circ} \mathrm{C}$, a relative humidity of $60 \pm 10 \%$, and a natural light-dark photoperiod.

\subsection{Animal experimental design}

Constipation was induced in the animals by injecting $1 \mathrm{~mL}$ of $4 \mathrm{mg} / \mathrm{kg}$ loperamide twice a day for 3 days, while the control rats were administered normal saline [11]. Successful modeling was performed at day 0 (D0).

AABW was given at a dose of ( $20 \mathrm{mg} / \mathrm{kg}, 40 \mathrm{mg} / \mathrm{kg}$ and $80 \mathrm{mg} / \mathrm{kg}$, b. w., p. o.) for 3 days (D1-D3) after modeling success. Other groups were treated with $1.2 \mathrm{~g} / \mathrm{kg}$ Maren Pills and $5.0 \mathrm{mg} / \mathrm{kg}$ Mosapride, which were used as the positive control drugs [12].

\subsection{Gastrointestinal function test}

After successful modeling and completion of treatment, feces of rats in each group were collected within $2 \mathrm{~h}$, weighed by electronic balance, and the quantity, wet weight and water content of feces of rats were recorded. GIP transit was measured through gavage activated carbon suspension $2 \mathrm{~mL}$ (concentration: $100 \mathrm{~g} / \mathrm{L}$ ) [13]. The gastrointestinal propulsion was calculated based on the following formula:

GIP $=($ Distance travelled by charcoal $(\mathrm{cm}) /$ total intestinal length $(\mathrm{cm})) \times 100 \%$

\subsection{Histopathological analysis}

Paraffin sections of $5 \mu \mathrm{m}$ thick were taken from the colon of rats. The sections were dewaxed in xylene and then dehydrated in gradient ethanol. After rehydration, colon tissue sections were stained with $\mathrm{HE}$ according to the instructions of HE staining kit, and the morphological changes of colon tissue were observed under a Olympus BX60 research microscope (Olympus Corporation, Tokyo, Japan) [11].

\subsection{Elisa analysis}


After blood samples were extracted from the abdominal aorta, the upper serum was collected by centrifugation at low temperature. Corresponding ELISA kit was used to detect the contents of NO, VIP,MTL, Gas, SP and 5-HT in peripheral blood [14].

\subsection{Western blot analysis}

After conventional homogenization, total protein was extracted from colon tissue with RIPA lysate. Protein quantification was performed using the BCA kit. $30 \mu$ g protein was taken from each sample, denatured by boiling, and 10\% SDS-PAGE was performed. The electrophoretic protein was electrotransferred to PVDF membrane. After transfer, the membrane was sealed with $5 \%$ skim milk powder at room temperature for $1 \mathrm{~h}$, followed by primary antibody (SCF: AB_1542939, c-Kit: AB_10618857, AQP3: AB_11188824 and AQP8: AB_800001, 1:1000), and incubated at $4{ }^{\circ} \mathrm{C}$ overnight. The next day, after washing with TBST, the secondary IRDye ${ }^{\circledR} 800 \mathrm{CW}$ Goat anti-Rabbit/Mouse $\lg \mathrm{G}(\mathrm{H}+\mathrm{L})$ antibody was added and incubated at room temperature for $1.5 \mathrm{~h}$. After washing with PBST, the blots were developed using an Odyssey ${ }^{\circledR} \mathrm{CLx}$ Imaging System with image lab software $[15,16]$.

\subsection{Real-time quantitative $P C R$ analysis}

The colon tissue was removed from the RNA Later liquid and the total RNA was extracted by Trizol method. Primer Premier3.0 software was used to design the PCR primers, and primer sequences were shown in Table 1. RNA from colon tissue was extracted according to the experimental procedure provided by the reagent description. After the total RNA was successfully extracted, $2 \mu \mathrm{L}$ RNA was added into 98 $\mu \mathrm{L}$ DEPC water, and the absorbance (OD) at $260 \mathrm{~nm}$ and $280 \mathrm{~nm}$ was determined on NanoDrop ND-8000 (Thermo, Waltham, MA, USA) instrument, with a ratio of $1.8 \sim 2$. Between $0.20 \mu \mathrm{L} \mathrm{cDNA}$ was obtained by reverse transcription of $1 \mu \mathrm{g}$ RNA. According to the instructions of real-time quantitative PCR kit, $1 \mu \mathrm{L}$ cDNA was configured into a $10 \mu \mathrm{L}$ reaction system. The reaction conditions were pre-denaturation at $95^{\circ} \mathrm{C}$ for $10 \mathrm{~min}, 95^{\circ} \mathrm{C}$ for $15 \mathrm{~s}, 60^{\circ} \mathrm{C}$ for $1 \mathrm{~min}$, and cycling 40 times. Finally, the dissolution curve program was added $\left(95^{\circ} \mathrm{C}\right.$ for $15 \mathrm{~s}, 60^{\circ} \mathrm{C}$ for $1 \mathrm{~min}$, and $95^{\circ} \mathrm{C}$ for $15 \mathrm{~s}$ ). Thresholdcycles (Ct) of the sample was obtained by using the RQ Manager data analysis software to calculate the relative copy levels and then compare the samples. Ct values measured using $2^{-\Delta \Delta C t}$ method [17]. 
Table 1

primers sequences

\begin{tabular}{|ll|}
\hline Indicators & primers sequences \\
\hline SCF & F: 5'-CTC AGT TTT GTG GCT TCG TTT A-3' \\
\hline C-Kit & R: 5'-CTA CCA TGT CCG ATA CTA CGA C-3' \\
\hline FQP3 $5^{\prime}-C A G$ AAA CCC ATG TAT GAA GT-3' & R: 5'-CTT TCC AAA ACT CAG CCT GT-3' \\
& F: 5'-GCC ATT GTT GAC CCT TAT AAC AAC-3' \\
AQP8 & F: 5'-CTG TGT GTA TGG GTG CCG TCA A-3' \\
\hline PGE2 & R: 5' F'AGA TCC CAC CAC CTG CCA GGA-3' $^{\prime}$-CTC GGC TGC AAC ATC AGT GT-3' \\
\hline GAPDH & F: 5' 5'-AGT CCT TTC TCC TCT CCG GC-3' \\
\hline & R: 5'-GGC ATG GAC TGT GGT CAT GA-3' \\
\hline
\end{tabular}

\subsection{Statistical analysis}

All data were subjected to a one-way ANOVA and a Dunnett's T test and the data were expressed as the mean \pm SEM by SPSS 20.0. $P$ values less than 0.05 or 0.01 indicated significant or extremely significant differences, respectively, and these differences were labeled with * or ** (extremely significant difference).

\section{Results}

\subsection{Effect of AABW on stool parameters}

Compared with the control group, loperamide significantly reduced the fecal quantity, wet weight and water content of rats. AABW could improve this abnormal change, as shown in Fig. 2.

\subsection{Effect of AABW on small intestinal transit rates (GIP)}

Compared with the control group, the GIP of the model group was significantly lower $(P<0.01)$. Compared with the model group, AABW $80 \mathrm{mg} / \mathrm{kg}$ could improve or even restore intestinal peristalsis in Fig. 3.

\subsection{Effects on Histopathology}


In the normal group, the colonic mucosa was intact, and the four layers of the mucosal layer, submucosa, muscular layer and outer layer of the intestinal wall were clear, the columnar cells were arranged neatly, and the goblet cells were mixed in the middle. No inflammatory cells were found and the glands were arranged neatly. In the model group, a large number of inflammatory cells infiltrated, interstitial vasodilation and hyperemia; AABW $80 \mathrm{mg} / \mathrm{kg}$ and positive control groups showed obvious improvement of inflammatory cell infiltration, and intestinal mucosa was arranged neatly in Fig. 4.

\subsection{Gastrointestinal hormone levels}

Compared with the control group, loperamide significantly decreased levels of Gas, MTL, SP, 5-HT and VIP and noticeably increased the content of NO $(P<0.05, P<0.01)$. Compared with the model group, treatments with Maren Pills, Mosapride and AABW $80 \mathrm{mg} / \mathrm{kg}$ were shown to improve abnormal levels of gastrointestinal hormones (Fig. 5) $(P<0.05, P<0.01)$.

\subsection{Expressions of SCF and c-Kit, AQP3 and AQP8 proteins}

Compared with the normal group, the expression levels of SCF and c-Kit protein in the model group were significantly lower; compared with the model group, the expression levels of positive control groups and AABW $80 \mathrm{mg} / \mathrm{kg}$ were significantly increased (Fig. 6).

Compared with the normal group, the expression of AQP3 in the constipation group clearly decreased; however, constipation rats treated with AABW $80 \mathrm{mg} / \mathrm{kg}$ showed an increase in AQP3 expression. Nonetheless, the expression of AQP8 was opposite to that of AQP3 (Fig. 6).

\subsection{SCF, c-Kit, VIP, AQP3 and AQP8 mRNA expression levels of the constipation-related genes}

As shown in results, the levels of mRNA expression of SCF, c-Kit, AQP3 and VIP were significantly downregulated, and the level of mRNA expression of AQP8 was significantly upregulated in the loperamide-induced rat colon $(P<0.01)$. However, the levels of mRNA expression in the AABW-treated constipation rat groups showed that AABW could significantly enhance the mRNA expression levels of SCF, C-Kit, AQP3 and VIP, and AABW could significantly decrease the mRNA expression level of AQP8 $(P<$ $0.05, P<0.01$ ) (Fig. 7).

\section{Discussion}

STC is the most common type of chronic functional constipation, which has a high incidence in the population and is difficult to treat. STC has become one of the important factors affecting people's quality of life worldwide. Data show that the prevalence of constipation in Chinese adult population is $3 \%$ $\sim 17 \%$, which is higher in females, 4 times as high as that in males $[18,19]$. AABW, as a component from Anemarrhena asphodeloides Bge., has been reported to play multiple effects, including relieving 
constipation. However, the detailed mechanisms through which AABW exerted laxative effects remain to be elucidated. The results of this study showed that AABW could promote defecation and increase fecal water content in constipated rats, and reduce fecal passage time in the intestine. These results suggested that AABW has the effect of relieving constipation (Fig. 2-3).

Loperamide directly stimulates enteric-wall $\mu$-receptors, inhibits the production of Ache and PEG2, and reduces intestinal peristalsis and secretion of gastrointestinal hormones, thus playing an anti-diarrhea role [20]. The rat constipation model induced by loperamide has a short establishment period and a long duration of symptoms, and can well simulate the pathophysiological characteristics of constipation. It should be pointed out that gender had a great influence on the establishment of the constipation model, and female rats had a more obvious difference in modeling effect. In order to avoid the interference of unknown factors, male rats were finally selected [21, 22]. In this study, it was found that rats were injected with loperamide, combined with symptoms, colonic fecal volume and fecal water content, to preliminarily determine the establishment of loperamide induced constipation. Above all, it could be concluded that the rat model of constipation induced by lomeramide was successfully replicated (Fig. 2).

Constipation is associated with an enteric nervous System (ENS) disorder. In ENS, 5-hydroxytryptamine (5-HT), vasoactive intestinal peptide (VIP) and NO are all neurotransmitters related to peristaltic activity of gastrointestinal tract [23-25]. 5- $\mathrm{HT}$ is an excitatory neurotransmitter, which can directly act on the 5- $\mathrm{HT}_{4}$ receptor in the intestinal pheochromocytoma cells in the superior colonic mucosa to cause intestinal smooth muscle contraction and promote intestinal peristalsis to cause defecation [23]. VIP could promote gastric empties, promote digestive secretion, inhibit gastrointestinal smooth muscle relaxation, and play a promoting role in gastrointestinal movement $[24,29,30]$. MTL and Gas could promote the secretion of pepsin, contract gastrointestinal smooth muscle, promote gastric peristalsis, and play an exciting role in gastrointestinal movement [25]. NO is a non-specific inhibitory neurotransmitter in ENS. Excessive NO could be diffused into intestinal smooth muscle cells to reduce intracellular $\mathrm{Ca}^{2+}$ concentration, thus causing excessive relaxation of intestinal stage smooth muscle and excessive intestinal stage peristalsis (intestinal spasm) [26, 27]. AABW could improve the symptoms of constipation by relieving the above gastrointestinal hormone and neurotransmitter abnormalities (Fig. 5)

At present, the etiology of STC is not clear, but slow intestinal peristalsis and excessive absorption of intestinal water are two important factors for the occurrence of constipation. Recent studies have shown that ICC and AQPs are closely related to intestinal dynamics and intestinal fluid metabolism, respectively. ICC is closely related to intestinal peristalsis and is considered as a pacemaker cell of intestinal peristalsis, which can initiate rhythmic intestinal myoelectric activity and simultaneously produce contraction rhythm of gastrointestinal smooth muscle [30]. A study showed that the ICC density of constipation patients was lower than that of normal people, and the decrease of ICC density resulted in the loss of ICC spontaneous rhythmic slow wave effect and colonic motor disorder. C-Kit and its ligand SCF are the main factors for ICC growth, development and maintenance [31-34]. AABW significantly upregulated the levels of both c-Kit and SCF in mice with Lop-induced constipation (Fig. 6). These results suggested that AABW increased the numbers of ICCs in mice with Lop-induced constipation. 
Aquaporin (AQPs) is closely related to intestinal water absorption. Abnormal expression of AQPs in the intestinal tract will have an impact on intestinal hydrologic metabolism, and is closely related to the occurrence and development of constipation [35]. At present, 13 subtypes of AQP family have been found (AQP0-AQP12), among which AQP3 plays an important role in intestinal hydrohydration metabolism. VIP is a neuropeptide regulating intestinal smooth muscle contraction and intestinal fluid metabolism, while AQP3 is mainly distributed in human intestinal and colon epithelial cells, and plays an important role in intestinal epithelial cell fluid reabsorption. VIP might bind to the receptor of intestinal epithelial cells and regulate the expression of AQP3 in the intestine of rats through the CAMP-PKA signaling pathway [36]. Recent studies have found that a variety of inflammatory factors have regulation on AQP3, such as IL-6, PEG2. PEG2 is associated with increased AQP endocytosis and degradation [37]. Stimulating colon to release PEG2 and VIP and down-regulating AQP3 expression in epithelial cytoplasm may be the key link of AABW in regulating the water metabolism of hemp canal. By regulating the content of functional AQP3 in the plasma membrane of colonic epithelial cells, the efficiency of water transport can be regulated, which further influences the hydrologic metabolism of colon. This might be the biological basis for the role of AABW (Figs. 1D, 6 and 7).

\section{Conclusion}

In conclusion, AABW might improve intestinal water liquid metabolism by regulating the colon absorption and colon secretion of both ICCs and AQPs in the enteric nervous system, so that its function can be improved or even restored to normal, accelerate colonic peristalsis, promote fecal discharge, relieve and treat constipation symptoms.

\section{List of abbreviations}

\begin{tabular}{|llcl|}
\hline HPLC & \multicolumn{4}{l|}{ High Performance Liquid Chromatography } & \\
\hline UV & Ultraviolet Rays & NO & Nitric oxide \\
p.o. & per os & HE & Hematoxylin-eosin \\
ELISA & enzyme linked immunosorbent assay & ICC & interstitial cells of Cajal \\
IL-6 & Interleukin-6 & PEG2 & prostaglandin E2 \\
\hline
\end{tabular}

\section{Declarations}

\section{Declarations}

\section{Ethics approval and consent to participate}

All animal experiments were implemented according to the Animal Care Committee of the School of Medicine, Heilongjiang University of Chinese Medicine (Approval Number, 2017050801). 


\section{Consent for publication}

All authors listed have approved the publication of this manuscript.

\section{Availability of data and materials}

The research data generated from this study is included within the article.

\section{Competing interests}

The authors of the paper have no financial or personal relationships with other people or organizations that would create a conflict of interest.

\section{Funding}

Our work was supported by the Major State Basic Research Development Program (973 Program) of China (2013CB531801), National Key Research and Development Project(2018YFC1707100).

\section{Authors' contributions}

Contributions of Xiaomao Li and Yan Liu were equal for designing the experiment, conducting the experiment, processing the data and writing the manuscript. Yonggang Xia and Yanping Sun touched up the manuscript. Bingyou Yang and Haixue Kuang provided funding and supervised the experiment.

\section{Acknowledgements}

No applicable.

\section{References}

1. Suares NC, Ford AC. Prevalence of, and risk factors for, chronic idiopathic constipation in the community: systematic review and meta-analysis. Am J Gastroenterol. 2011;106:1582-91.

2. Liu XM, Peng ZR, Ni XQ, Yang J, Zou ZY, Qiu CH, Zeng D. L. G. Yang. Aperient effect of fructooligosaccharides and lactobacillus on constipation model of rats. Food Science. 2013;34:2969.

3. Liang LN, Fan XQ, Yu ZG, Lu SM, Liu LN, Li CY. Therapeutic effects of lactulose in combination with live combined Bacillus subtilis and Enterococcus faecium enteric-coated capsules in elderly patients with chronic functional constipation. World Chinese Journal of Digestology. 2016;24:316-21.

4. Ke M. Confusion and misunderstanding in the diagnosis and treatment of chronic constipation. National Medical Journal of China. 2007;87:649-50. 
5. Ma JZ, Liu SN, Wu TX, Shi L. Efficacy of Chinese herbal medicines in treating chronic functional constipation: a systematic review. Chin J Evid-based Med. 2010;10:1213-21.

6. Kim JE, Go J, Koh EK, Song SH, Sung JE, Lee HA, et al. Gallotannin-enriched extract isolated from Galla Rhois may be a functional candidate with laxative effects for treatment of loperamide-induced constipation of SD rats. Plos One. 2016;11:1-17.

7. Han SH, Park K, Kim EY, Ahn SH, Lee HS. H. J. Suh. Cactus (Opuntia humifusa) water extract ameliorates loperamide-induced constipation in rats. BMC Complementary Alternative Medicine. 2017;17:1-8.

8. Jabri MA, Wannes D, Hajji N, Sakly M, Marzouki L. H. Sebai. Role of laxative and antioxidant properties of Malva sylvestris leaves in constipation treatment. Biomed Pharmacother. 2017;89:2935.

9. Luo DD, Qu C, Lin GS, Zhang ZB, et al. Character and laxative activity of polysaccharides isolated from Dendrobium officinale. Journal of Functional Food. 2017;34:106-17.

10. Zhang YG, Shao WJ, F.Gu Y, Qiu JF, Yuan L, Li GD. Effects of sacral nerve stimulation with acupuncture on gut transit time and c-Kit expression in colon of rats with slow transit constipation. Genet Mol Res. 2016;15:1-8.

11. Zhang WP, Jing B, Ding SQ, Xu S, Huangfu SH. Effect of colonic bypass with ileorectal anastomosis on plasma levels of SP and VIP in rats with slow transit constipation. World Chinese Journal of Digestology. 2012;20:585-9.

12. Lu CL, Zhu W, Wang M, et al. Polysaccharides from Smilax glabra inhibit the pro-inflammatory mediators via ERK1/2 and JNK pathways in LPS-induced RAW264.7 cells. Carbohyd Polym. 2015;122:428-36.

13. Du LJ, Zhan Y, Wu ZJ, et al. Effect of Rhubarb on expression of aquaporin - 3 mRNA and motility in colon Tissues of rats with constipation, Chinese archives of traditional Chinese medicine. 2017; 35 : 873-875.

14. Mugie SM, Benninga MA. L. C. Di. Epidemiology of constipation in children and adults: a systematic review. Best Pract Res Clin Gastroenterol. 2011;25:3-18.

15. Ke Huang Li-an, Li Yuan-guang, Meng Yan-qin, You Xiao-yu, Fu L Song. Arctigenin promotes apoptosis in ovarian cancer cells via the iNOS/NO/STAT3/survivin signalling. Basic \& Clinical Pharmacology \& Toxicology. 2014; 115: 507-511.

16. Braak B, Klooker TK, Wouters MM, Welting O. C. M. Van. Mucosal immune cell numbers and visceral sensitivity in patients with irritable bowel syndrome: is there any relationship? Am J Gastroenterol. 2012;107:715-26.

17. Frattini JC, Nogueras JJ. Slow transit constipation: a review of a colonic functional disorder. Clinics in Colon Rectal Surgery. 2008;21:146.

18. Tomita R, Igarashi S, Fujisaki S, Tanjoh K. The effects of neurotensin in the colon of patients with slow transit constipation. Hepato-gastroenterology. 2007;54:1662. 
19. Zhu YF, Wang XY, Lowie BJ, et al. Enteric sensory neurons communicate with interstitial cells of Cajal to affect pacemaker activity in the small intestine. Pflügers Archiv - European Journal of Physiology. 2014;466:1467-75.

20. Camborová P, Hubka P, Sulková I, Hulín I. The pacemaker activity of interstitial cells of Cajal and gastric electrical activity. Physiol Res. 2003;52:275.

21. Maria-Simonetta FP, Camillo C, Paolo R. The ultrastructure of the muscle coat of human gastrooesophageal junction, with special reference to "interstitial cells of Cajal". Frontiers in Neuroscience. 2013;7:49.

22. Kashyap P, Gomezpinilla PJ, Pozo MJ, Cima RR. E. J. Dozois. Immunoreactivity for Ano1 Detects Depletion of Kit-positive Interstitial Cells of Cajal in Patients with Slow Transit Constipation. Neurogastroenterology Motility. 2011;23:760-5.

23. Jonathan Y, Stephen K, Wu Q, Borg JF, Natalia L. Characterization of AQPs in Mouse, Rat, and Human Colon and Their Selective Regulation by Bile Acids. Frontiers in Nutrition. 2016;3:46-51.

24. Chao G, Zhang S. Aquaporins 1, 3 and 8 expression in irritable bowel syndrome rats' colon via NF-kB pathway. Oncotarget. 2017;8:47175-83.

25. Ikarashi N, Mimura A, Kon R, lizasa T, Omodaka M. The concomitant use of an osmotic laxative, magnesium sulphate, and a stimulant laxative, bisacodyl, does not enhance the laxative effect. Eur $\mathrm{J}$ Pharm Sci. 2012;45:73.

26. Ikarashi N, Ushiki T, Mochizuki T, Toda T, Kudo T. Effects of magnesium sulphate administration on aquaporin 3 in rat gastrointestinal tract. Biological Pharmaceutical Bulletin. 2011;34:238.

27. Zhao GX, Dong PP, Peng R, Li J. D. Y. Zhang. Expression, localization and possible functions of aquaporins 3 and 8 in rat digestive system. Biotech Histochem. 2016;91:269-76.

28. Danil I, Peregud AA, Yakovlev MYu, Stepanichev MV, Onufriev, Leonid F, Panchenko NV. Gulyaeva. Expression of BDNF and TrkB phosphorylation in the rat frontal cortex during morphine withdrawal are NO dependent. Cell Mol Neurobiol. 2015;36:839-49.

29. Tomita R, Igarashi S, Fujisaki S, Tanjoh K. The effects of neurotensin in the colon of patients with slow transit constipation. Hepato-gastroenterology. 2007;54:1662-6.

30. Xu J, Chen Y, Liu S, Hou X. Electroacupuncture regulates apoptosis/proliferation of intramuscular interstitial cells of cajal and restores colonic motility in diabetic constipation rats. Evid Based Complement Alternat Med. 2013;18:584179.

31. Farrugia G. Interstitial cells of Cajal in health and disease. Neurogastroenterol Motil. 2008;20:54-63.

32. Chang S, Yu HC, Kim HK, Hong, et al. Evaluation of myenteric ganglion cells and interstitial cells of Cajal in patients with chronic idiopathic constipation. Int J Colorectal Dis. 2002;17:253-8.

33. Mostafa RM, Moustafa YM, Hamdy H. Interstitial cells of Cajal, the Maestro in health and disease. World J Gastroenterol. 2010;16:3239-48.

34. Parthasarathy G, Chen J, Chia N, O'Connor HM, Gaskins HR, Bharucha AE. Reproducibility of assessing fecal microbiota in chronic constipation. Neurogastroenterol Motil. 2017;29:1-10. 
35. Silberstein C, Kierbel A, Amodeo G, et al. Functional characterization and localization of AQP3 in the human colon. Braz J Med Biol Res. 1999;32:1303-13.

36. Risako Kon N, Ikarashi A, Hayakawa, et al. Morphine-induced constipation develops with increased aquaporin-3 expression in the colon via increased serotonin secretion. Toxicol. 2015;145:337-47.

37. Nobutomo Ikarashi A, Mimura R, Kon, et al. The concomitant use of an osmotic laxative, magnesium sulphate, and a stimulant laxative, bisacodyl, does not enhance the laxative effect. Eur J Pharm Sci. 2012;45:73-8.

\section{Figures}
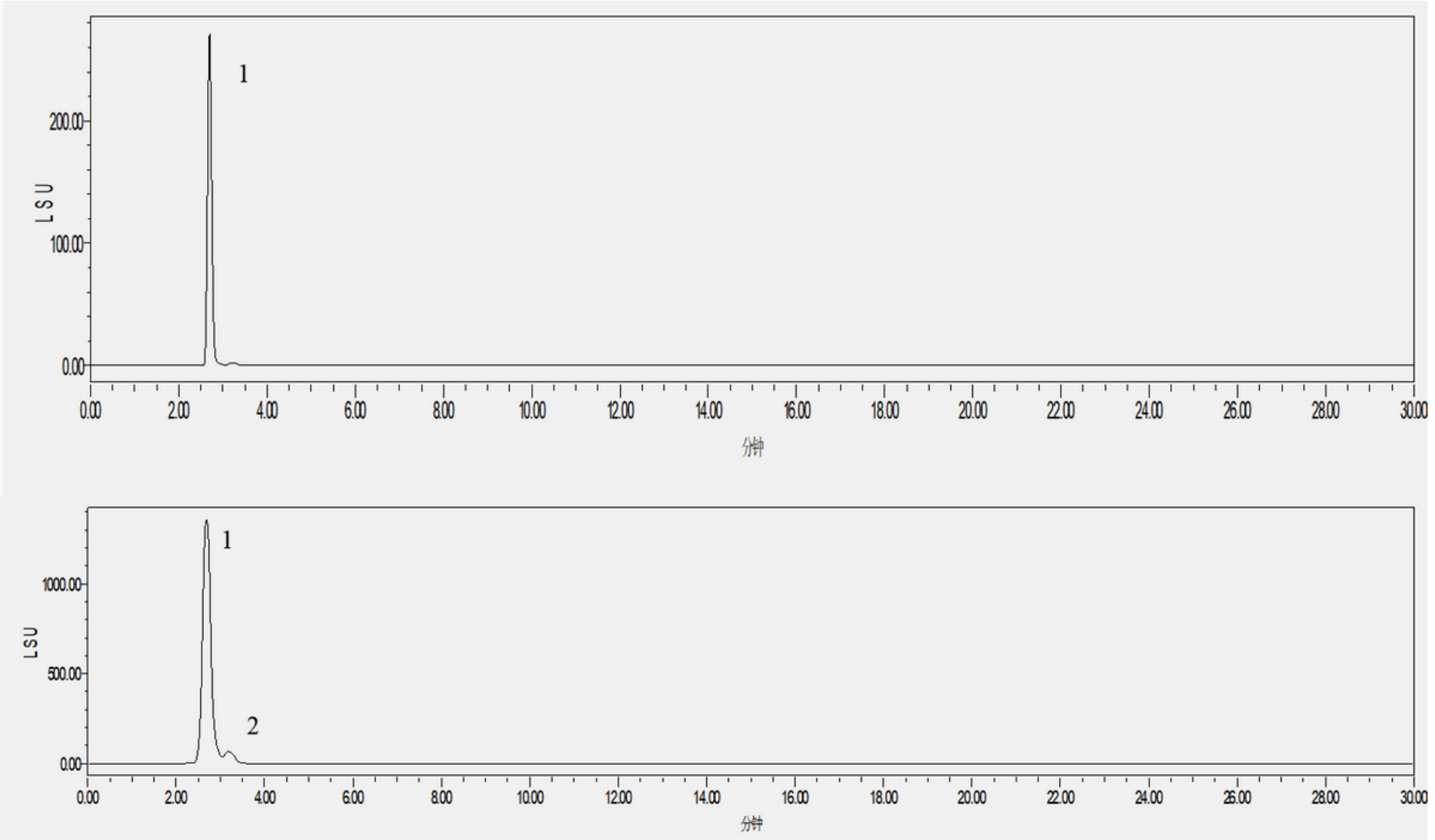

\section{Figure 1}

HPLC analysis of AABW and fructose standard 

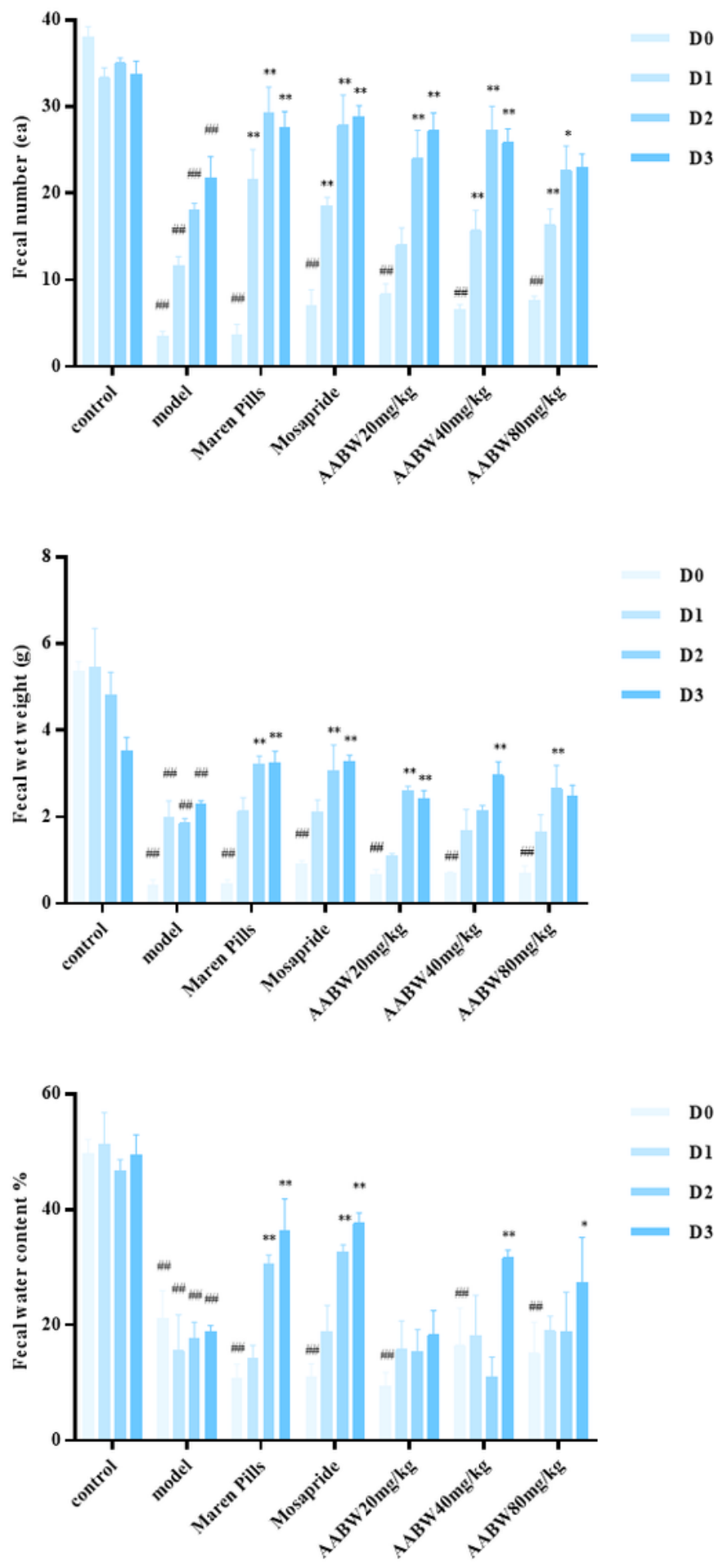

Figure 2

Effects of AABW on faecal output character in loperamide-induced rats. (A) number of faeces; (B) wet weight of faeces; (C) water content. Mean \pm SEM, $n=8$ \#\#P< 0.01 compared to the control group, while ${ }^{*} \mathrm{P}<0.05$ and ${ }^{* *}<0.01$ compared to loperamide-induced group. 


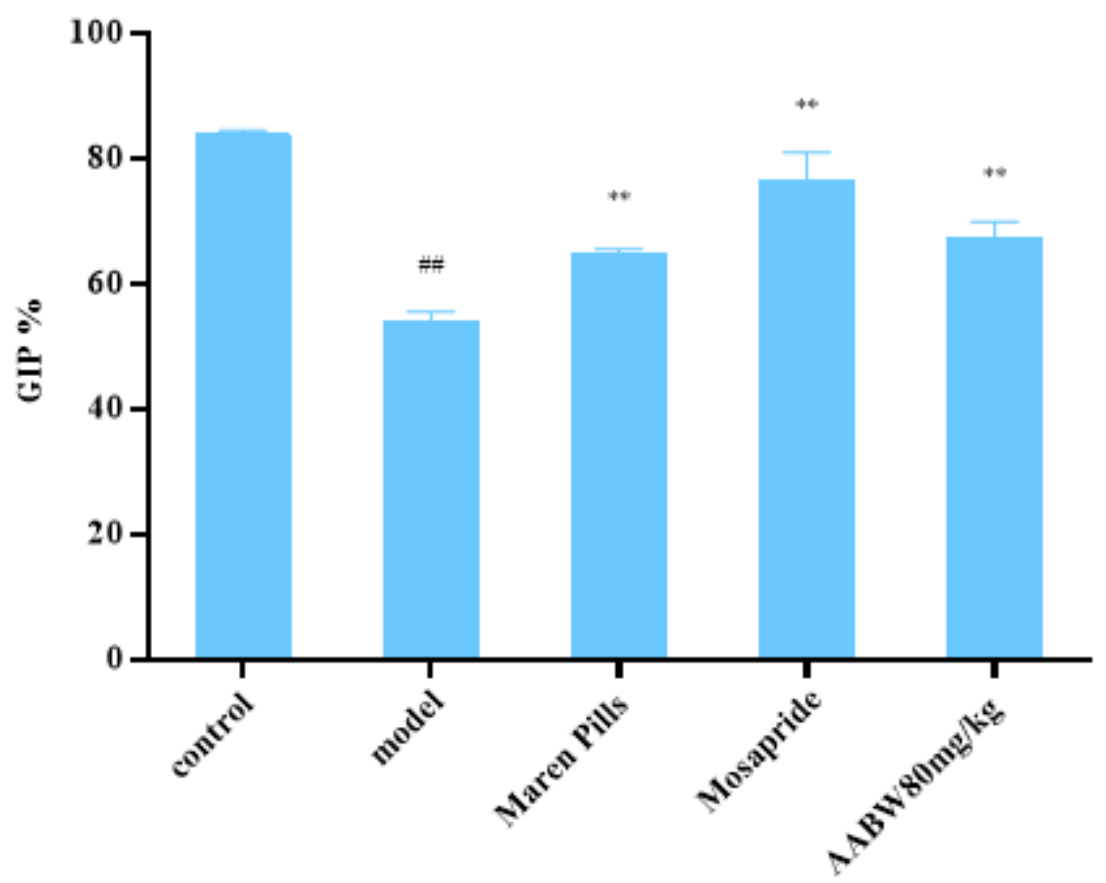

Figure 3

Small intestinal transit rate of rats. Mean \pm SEM, $n=8$ \#\#P $<0.01$ compared to the control group, while $\star \mathrm{P}<0.05$ and $* *<0.01$ compared to loperamide-induced group.

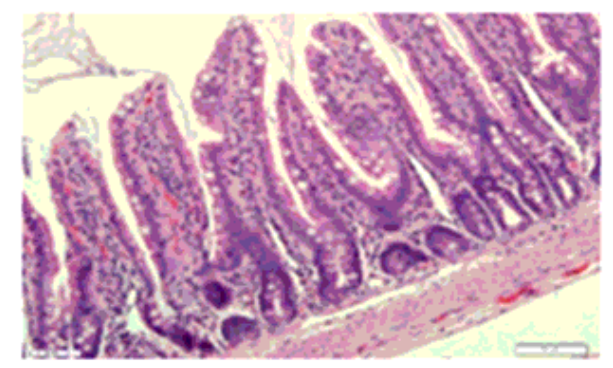

control

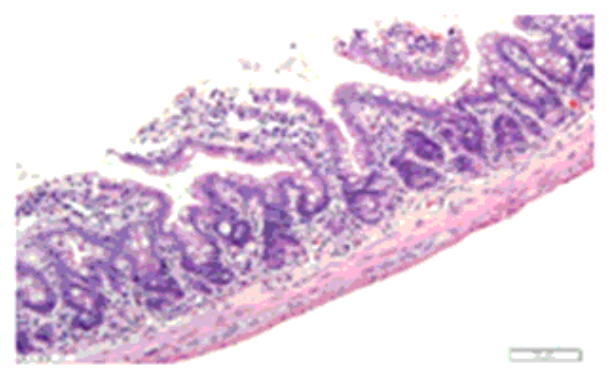

model

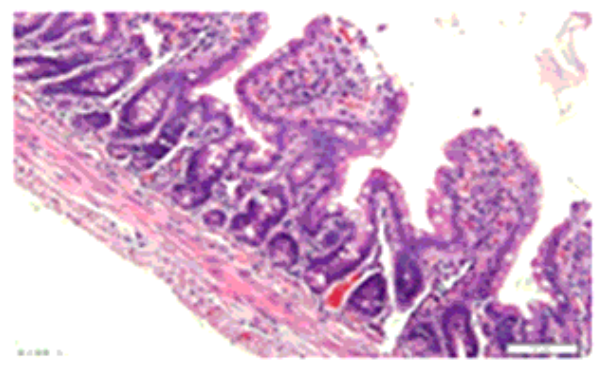

Maren Pills

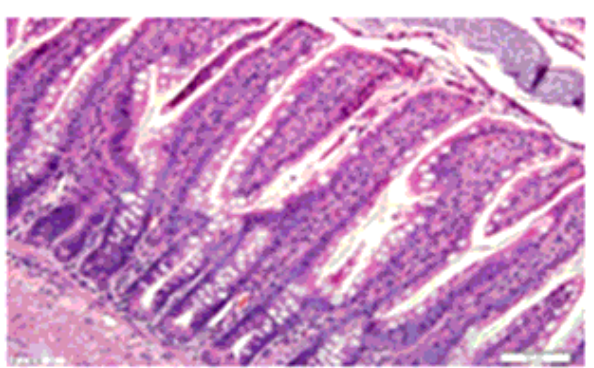

Mosapride

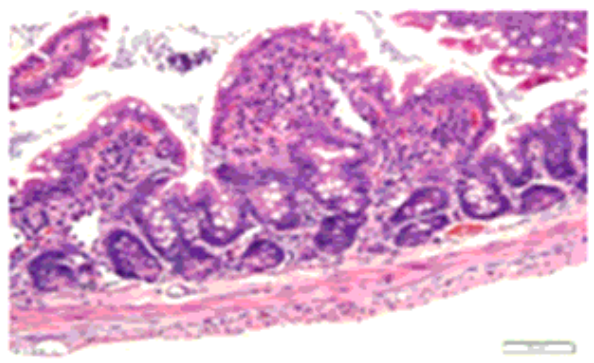

AABW80 mg/kg

Figure 4 
Representative slides of HE stain results for colon sections (magnification, $\times 200$ ).
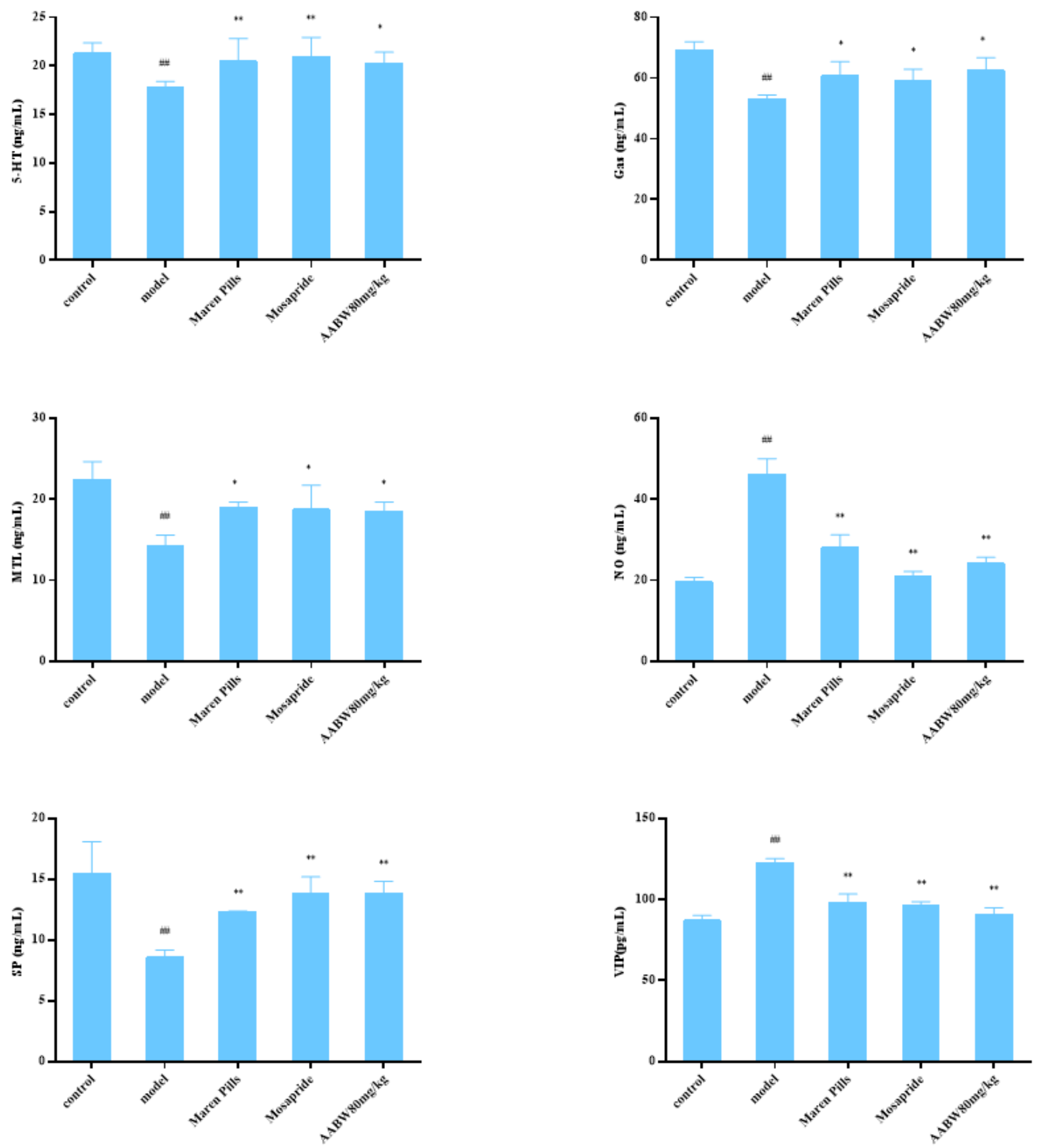

Figure 5

Effect of AABW on serum levels of Gas, MTL, SP, 5-HT, VIP and NO. Mean \pm SEM, $n=8 \# \# P<0.01$ compared to the control group, while ${ }^{*} \mathrm{P}<0.05$ and $* *<0.01$ compared to loperamide-induced group. 


\section{$\beta$-actin}

SCF

C-Kit

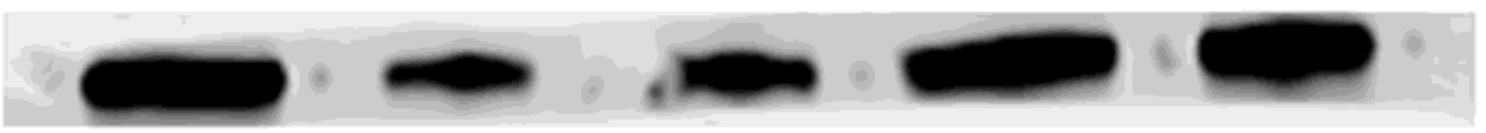

AQP3

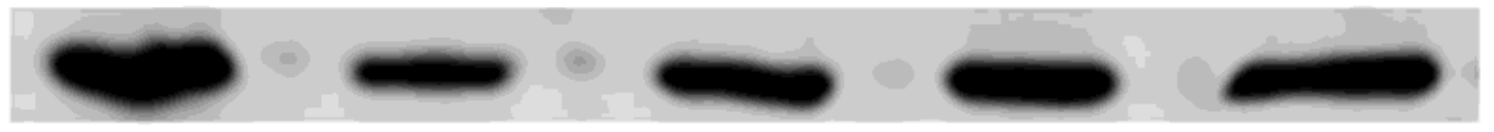

AQP8
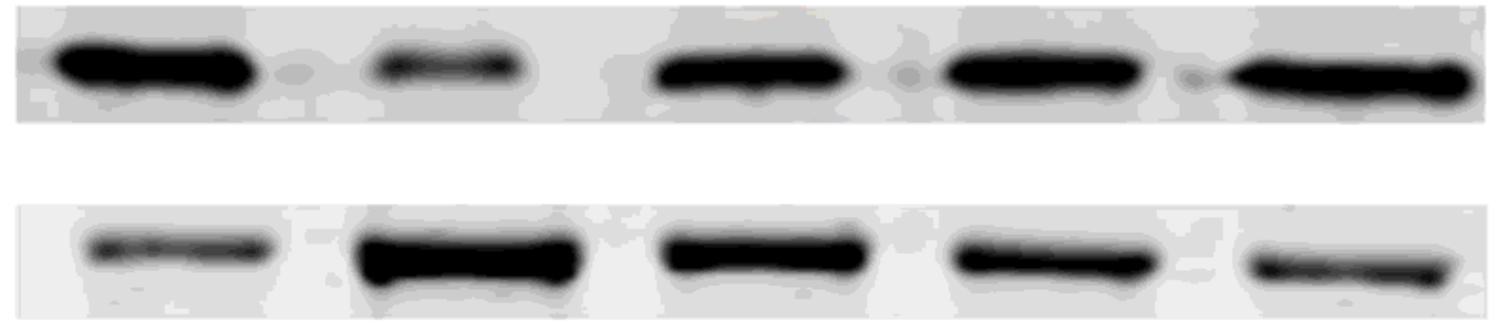

Figure 6

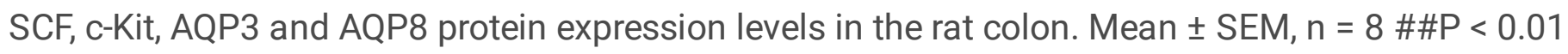
compared to the control group, while $* \mathrm{P}<0.05$ and ${ }^{*}<0.01$ compared to loperamide-induced group.

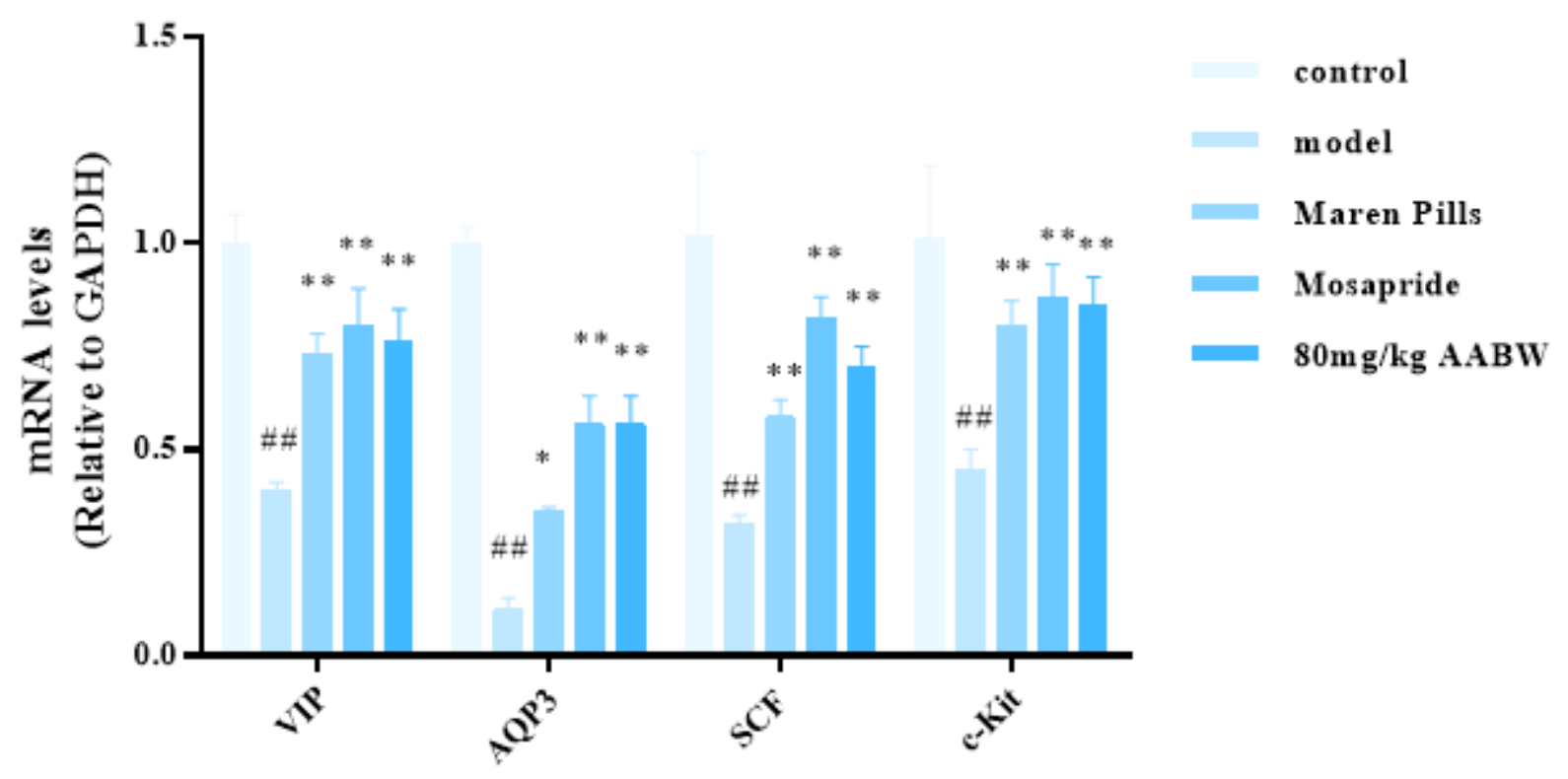

Figure 7

Effects of AABW on the mRNA levels of SCF, c-Kit, AQP3, VIP, in rat colons. Mean \pm SEM, $n=8 \# \# P<0.01$ compared to the control group, while ${ }^{*} \mathrm{P}<0.05$ and ${ }^{*}<0.01$ compared to loperamide-induced group. 